\title{
Paclitaxel chemotherapy: from empiricism to a mechanism-based formulation strategy
}

\author{
Charity D Scripture \\ William D Figg \\ Alex Sparreboom \\ Clinical Pharmacology Research \\ Core, National Cancer Institute, \\ Bethesda, MD, USA
}

\begin{abstract}
Paclitaxel is an anticancer agent effective for the treatment of breast, ovarian, lung, and head and neck cancer. Because of water insolubility, paclitaxel is formulated with the micelle-forming vehicle Cremophor EL to enhance drug solubility. However, the addition of Cremophor EL results in hypersensitivity reactions, neurotoxicity, and altered pharmacokinetics of paclitaxel. To circumvent these unfavorable effects resulting from the addition of Cremophor EL, efforts have been made to develop new delivery systems for paclitaxel administration. For example, ABI-007 is a Cremophor-free, albumin-stabilized, nanoparticle paclitaxel formulation that was found to have significantly less toxicity than Cremophor-containing paclitaxel in mice. Pharmacokinetic studies indicate that in contrast to Cremophor-containing paclitaxel, ABI-007 displays linear pharmacokinetics over the clinically relevant dose range of $135-300 \mathrm{mg} / \mathrm{m}^{2}$. In a phase III study conducted in patients with metastatic breast cancer, patients treated with ABI-007 achieved a significantly higher objective response rate and time to progression than those treated with Cremophor-containing paclitaxel. Together these findings suggest that nanoparticle albumin-bound paclitaxel may enable clinicians to administer paclitaxel at higher doses with less toxicity than is seen with Cremophor-containing paclitaxel. The role of this novel paclitaxel formulation in combination therapy with other antineoplastic agents needs to be determined.
\end{abstract}

Keywords: paclitaxel, nanoparticle albumin-bound paclitaxel, pharmacokinetics

\section{Introduction}

Paclitaxel is a hydrophobic antineoplastic agent demonstrating significant antitumor activity against a broad spectrum of human malignancies, including breast, lung, and ovarian cancer. Following the identification of paclitaxel as the active ingredient in crude ethanolic extracts of the bark of the Pacific yew tree, Taxus brevifolia, development of this drug was suspended for over a decade because of problems in drug formulation, drug supply, and controversies regarding the mechanism of action (Wani et al 1971). After investigation of a large variety of excipients to enable parenteral administration of paclitaxel, the formulation approach using the polyoxyethylated castor oil derivative Cremophor ${ }^{\circledR}$ EL $(\mathrm{CrEL})$ represented the most viable option (Adams et al 1993). Currently, paclitaxel is commercially marketed in a formulation that contains a solvent system of CrEL and dehydrated ethanol. CrEL is widely used as a vehicle for the solubilization of a number of other hydrophobic drugs including anesthetics, vitamins, sedatives, photosensitizers, immunosuppressants, and investigational anticancer drugs. The amount of CrEL in Taxol ${ }^{\circledR}$ per administration is relatively high, and therefore its toxicological and pharmacological behavior in the context of chemotherapeutic treatment with paclitaxel is of major importance (Gelderblom et al 2001; van Zuylen, Verweij, et al 2001).

The drawbacks presented by the presence of CrEL in the paclitaxel formulation have been an incentive for extensive research to develop alternative delivery forms. Currently, several strategies are in progress to develop a CrEL-free formulation of 
paclitaxel, including biological approaches (oral administration), chemical approaches (prodrugs, analogs), and pharmaceutical approaches (use of co-solvents, emulsions, liposomes, cyclodextrins, microspheres, nanoparticles) (Terwogt et al 1997; Nuijen et al 2001). The most extensively studied alternative paclitaxel preparation is ABI-007, a human albumin-stabilized, lyophilized nanoparticle formulation, with an average size of $130 \mathrm{~nm}$, which is free of CrEL and ethanol (Desai et al 2002). Comparative properties of this paclitaxel formulation and the conventional CrEL-containing formulation are highlighted in Table 1 and are discussed further.

\section{Preclinical studies of paclitaxel disposition}

In preclinical studies performed in mice, ABI-007 was shown to have significantly less toxicity than Taxol (Desai et al 2002). In five different human tumor xenografts (NCIH522 lung, MX-1 breast, SKOV-3 ovarian, PC-3 prostate, and HT29 colon), the maximum tolerated dose of ABI-007 was 1.5- to 2-fold greater than that of Taxol. In addition, at the maximum tolerated dose, ABI-007 showed superior efficacy to Taxol, especially in breast, colon, and ovarian models.

Plasma pharmacokinetics and partitioning of radiolabeled paclitaxel from ABI-007 and Taxol into red blood cells and tumor tissue was examined in an MX-1 xenograft tumor model over 24 hours following a single tail vein injection of paclitaxel $20 \mathrm{mg} / \mathrm{kg}$ (Desai et al 2003). ABI-007 distributed more rapidly and extensively than Taxol, as shown by a 5-fold larger volume of distribution, lower maximum concentration, and lower area under the plasma concentration-time curve (AUC), suggesting that CrEL prevented the distribution of Taxol out of the circulation and into the tissues. ABI-007 demonstrated a significantly lower plasma/blood ratio of paclitaxel $(\mathrm{p}<0.0001)$. ABI-007 also distributed more effectively into MX-1 tumor with a tumor AUC of paclitaxel 1.6-fold higher with ABI-007 than with Taxol $(\mathrm{p}<0.0001)$.

A series of preclinical studies and cell biology experiments have been conducted to investigate the mechanism of enhanced tumor penetration observed with ABI-007. A current hypothesis is that ABI-007 reaches the tumor by a novel mechanism (Desai et al 2004; Garber 2004). It has been suggested that some ABI-007 accesses the tumor via leaky junctions in the tumor vasculature and is retained by the tumor's impaired lymphatic system. Albumin has been shown to be transported across endothelial
Table I Comparative properties of nanoparticle albuminbound paclitaxel and Cremophor EL-containing paclitaxel

\begin{tabular}{lrr}
\hline & $\begin{array}{r}\text { Nanoparticle } \\
\text { albumin-bound } \\
\text { paclitaxel } \\
\text { (ABI-007) }\end{array}$ & $\begin{array}{r}\text { Cremophor } \\
\text { EL-based } \\
\text { paclitaxel } \\
\text { (eg,Taxol) }\end{array}$ \\
\hline $\begin{array}{l}\text { Hypersensitivity } \\
\text { reactions }\end{array}$ & No & Yes \\
$\begin{array}{l}\text { Leaches plasticizers } \\
\text { from intravenous tubing }\end{array}$ & No & Yes \\
$\begin{array}{l}\text { Forms micelles in plasma } \\
\text { Transported via gp60 } \\
\text { receptor on vascular } \\
\text { endothelial cells }\end{array}$ & No & Yes \\
Nonlinear pharmacokinetics & Yes & No \\
Drug-drug interactions & Not known & Yes \\
\hline
\end{tabular}

cells by a specific receptor (gp60)-mediated caveolar transport (John et al 2003). It was hypothesized that nanoparticle albumin-bound paclitaxel might be transported similarly, and increased ABI-007 transport by endothelial gp60-mediated transcytosis was recently reported (Desai et al 2004). This process has been shown to be selectively inhibited by Taxol (Desai et al 2004). This finding may partly explain the increased intratumoral concentrations of paclitaxel seen following ABI-007 administration relative to Taxol.

\section{Paclitaxel pharmacokinetics}

Various studies have shown that CrEL alters the pharmacokinetic behavior of many drugs administered intravenously, including cyclosporin, anthracyclines, etoposide, the irinotecan metabolite SN38, the photosensitizer $\mathrm{C} 8 \mathrm{KC}$, and paclitaxel. The most common effect is a substantial increase in systemic exposure to the studied agent with a concomitantly reduced systemic clearance, as was first described for paclitaxel in a mouse model. Various proposed causes of the CrEL-drug interactions have been put forward, including altered protein-binding characteristics (Sykes et al 1994), altered hepatobiliary secretion (Ellis and Webster 1999), and inhibition of endogenous P-glycoprotein-mediated biliary secretion, thereby reducing elimination of drugs (Gianni et al 1997). In the isolated perfused rat liver, CrEL inhibited the hepatic elimination of paclitaxel, preventing the drug from reaching the sites of metabolism and excretion (Ellis and Webster 1999). However, studies indicate that drugtransporting P-glycoproteins are not essential for normal hepatobiliary secretion of paclitaxel (Sparreboom et al 
1997), suggesting that this protein does not play a major role.

In view of the very small volume of distribution of CrEL, it is likely that the pharmacokinetic interaction observed with paclitaxel takes place within the central blood compartment. This was confirmed by in vitro experiments demonstrating that encapsulation of the model drug paclitaxel within the hydrophobic interior of CrEL micelles takes place in a concentration-dependent manner, causing changes in cellular partitioning and blood/plasma concentration ratios of paclitaxel (Sparreboom et al 1999; Loos et al 2002). It was shown that the affinity of paclitaxel was (in decreasing order) CrEL $>$ plasma $>$ human serum albumin, with CrEL present above the critical micellar concentration (ie, $\sim 0.01 \%$ ). Since the effect was also observed in the absence of plasma proteins, it could not have been caused by altered protein binding or by an increased affinity of paclitaxel for protein dissociation products that are produced by the action of CrEL on native lipoproteins (Sykes et al 1994). These findings are consistent with the hypothesis that paclitaxel can be entrapped within CrEL micelles, and that these micelles act as the principal carrier of paclitaxel in the systemic circulation.

An intriguing feature of Taxol pharmacokinetics is a distinct dose-dependent pharmacokinetic behavior, with clearance values decreasing substantially with an increase in drug dose. This effect is particularly evident with 3-hour infusion regimens, and CrEL has been linked to this phenomenon. It has been shown that the percentage of total paclitaxel trapped in micelles increases disproportionately with higher doses of CrEL administered (van Zuylen et al 2000; van Zuylen, Karlsson, et al 2001), thereby influencing the unbound drug concentration and making it less available for distribution to tumor, tissues, metabolism, and biliary and intestinal secretion. Indeed, the free fraction of paclitaxel is inversely related to $\mathrm{CrEL}$ concentrations in vitro (Brouwer et al 2000), and CrEL has also been shown to alter the blood/plasma concentration ratios in vivo by reducing drug uptake in red blood cells (van Zuylen, Karlsson, et al 2001). Interestingly, when paclitaxel dissolved in another vehicle was administered to mice, no pharmacokinetic nonlinearity in plasma concentration profiles was evident (Sparreboom et al 1996a, 1996b). The concentrations in tissues also increased linearly with escalating doses even when dissolved in CrEL, suggesting linear kinetics for the unbound drug. The nonlinearity in paclitaxel pharmacokinetics was previously described using empirical models using both saturable elimination and distribution, where the saturable distribution has been described as saturable transport (Sonnichsen et al 1994) or saturable binding (Karlsson et al 1999). A recent study demonstrated that a mechanistic model could be used to describe the nonlinear kinetics of the drug using simultaneous description of total and unbound plasma concentrations, whole blood concentrations, and concomitant CrEL levels (Henningsson et al 2001). This pharmacokinetic model has a foundation in the known properties of paclitaxel as determined with micellar trapping of paclitaxel, distribution to red blood cells, and binding to serum albumin, $\alpha_{1}$-acid glycoprotein, and platelets. The results of that study showed that the nonlinear pharmacokinetics of paclitaxel are predominantly explained by nonlinear binding to CrEL and that the unbound drug displayed linear pharmacokinetics when administered over a 3-hour period.

The drug fraction not bound to serum proteins or CrEL is a rather small fraction of the total under normal physiological conditions, and at high concentrations, paclitaxel is mainly bound to CrEL. From simulated concentration components in patients treated with 24-hour infusions in which CrEL concentrations are rather low, it was demonstrated that the linear paclitaxel binding to serum proteins and binding to blood cells are of greater importance than the CrEL binding (Henningsson et al 2001). The schedule-dependent clearance of CrEL has a serious clinical ramification, in that systemic exposure to unbound paclitaxel is a function of infusion duration. This was confirmed in a randomized, comparative clinical trial evaluating drug disposition characteristics following 1-versus 3-hour infusions (Gelderblom et al 2002). The AUC of unbound paclitaxel after a 1-hour infusion was $24 \%(p=0.009)$ less than the AUC demonstrated by the 3-hour infusion group, despite significantly higher peak concentrations associated with the shorter infusion time $(0.26 \pm 0.007$ vs $0.15 \pm 0.07 \mu \mathrm{mol} / \mathrm{L} ; \mathrm{p}=0.0002)$. Most importantly, this effect translated into more severe hematological toxicity with the 3-hour schedule of drug administration (Gelderblom et al 2002), suggesting that the various infusion schedules currently employed for paclitaxel dosing are neither interchangeable nor pharmacologically equivalent.

Paclitaxel pharmacokinetics following ABI-007 administration have been studied in 16 patients who received doses ranging from 135 to $375 \mathrm{mg} / \mathrm{m}^{2}$ using noncompartmental analysis (Ibrahim et al 2002; Hawkins et al 2004). Pharmacokinetic parameters are summarized in Table 2. Elimination of paclitaxel appears to be biphasic and near dose-proportional up to a dose of $300 \mathrm{mg} / \mathrm{m}^{2}$; a 
Table 2 Summary of noncompartmental pharmacokinetic parameters for paclitaxel following administration of ABI-007; data are presented as mean values (\% coefficient of variation)

\begin{tabular}{|c|c|c|c|c|c|c|c|}
\hline $\begin{array}{l}\text { Dose } \\
\left(\mathrm{mg} / \mathrm{m}^{2}\right)\end{array}$ & $\begin{array}{r}\text { Infusion } \\
\text { duration } \\
\text { (min) }\end{array}$ & $\begin{array}{r}\text { Number of } \\
\text { patients }\end{array}$ & $\begin{array}{r}C_{\max } \\
(n g / m L)\end{array}$ & $\begin{array}{r}\text { AUC }_{\infty} \\
(\mathrm{ng} \cdot \mathrm{h} / \mathrm{mL})\end{array}$ & $\begin{array}{r}\text { Half-life } \\
\text { (h) }\end{array}$ & $\begin{array}{r}C L \\
\left(L / h \cdot m^{2}\right)\end{array}$ & $\begin{array}{r}V_{z} \\
\left(L / m^{2}\right)\end{array}$ \\
\hline 135 & 30 & 1 & 6100 & 6427 & 14.6 & 21.1 & 442 \\
\hline 200 & 30 & 3 & $7757 \quad(35)$ & $9613 \quad(20)$ & 13.4 (67) & $2 \mathrm{I} .4 \quad(2 \mathrm{I})$ & $384 \quad(64)$ \\
\hline 260 & 30 & 14 & $22969(113)$ & 14789 & $21.6(17.2)$ & $21.1(43.8)$ & $663.8(48.1)$ \\
\hline 300 & 30 & 5 & 13520 & $17610 \quad(21)$ & 14.6 (14) & I7.7 (22) & $370 \quad(23)$ \\
\hline 375 & 30 & 4 & $19350 \quad(15)$ & $35805 \quad(40)$ & $13.2(12)$ & 11.9 & $236 \quad(54)$ \\
\hline
\end{tabular}

Adapted from Hawkins et al (2004) and Ibrahim et al (2002).

Abbreviations: $C_{\max }$, peak concentration; $A \cup C_{\infty}$, area under the concentration-time curve from time 0 to infinity; $C L$, total body clearance; $V_{z}$, volume of distribution.

2.2-fold increase in dose (from 135 to $300 \mathrm{mg} / \mathrm{m}^{2}$ ) is associated with a 2.2-fold increase in peak concentration and a 2.7 -fold increase in AUC. At a dose of $375 \mathrm{mg} / \mathrm{m}^{2}$, the clearance of paclitaxel decreased slightly, possibly suggesting nonlinear disposition pathways. The clinical relevance of this observation is unclear because few patients were treated at doses above $300 \mathrm{mg} / \mathrm{m}^{2}$ and the recommended dose is well below those associated with potential nonlinear pharmacokinetics. It is, however, significant that ABI-007 displays linear pharmacokinetics over the clinically relevant dose range of $135-300 \mathrm{mg} / \mathrm{m}^{2}$; over a similar dose range, Taxol $\mathrm{AUC}_{\infty}$ is nonlinear (Gianni et al 1995; Kearns 1997; Mross et al 2000).

The pharmacokinetics of paclitaxel administered as ABI007 or Taxol were recently compared (Hawkins et al 2004). Pharmacokinetic parameters are summarized in Table 3. Patients with advanced solid tumors were randomized to receive either ABI-007 $260 \mathrm{mg} / \mathrm{m}^{2}$ over 0.5 hour or Taxol $175 \mathrm{mg} / \mathrm{m}^{2}$ over 3 hours. As expected, owing to the difference in the dose of paclitaxel administered and the duration of infusion, statistically significant differences in

Table 3 Summary of pharmacokinetic parameters for paclitaxel following administration of $\mathrm{ABI}-007$ and Taxol; data are presented as mean values (\% coefficient of variation)

\begin{tabular}{|c|c|c|c|}
\hline Parameter & $\begin{array}{r}\text { ABI-007 } \\
260 \mathrm{mg} / \mathrm{m}^{2}\end{array}$ & $\begin{array}{r}\text { Taxol } \\
175 \mathrm{mg} / \mathrm{m}^{2}\end{array}$ & p-value \\
\hline $\mathrm{CL}\left(\mathrm{L} / \mathrm{h} \cdot \mathrm{m}^{2}\right)$ & 21.13 & 14.76 (3I.8) & 0.048 \\
\hline $\mathrm{Vd}_{\mathrm{ss}}\left(\mathrm{L} / \mathrm{m}^{2}\right)$ & $230.7 \quad(54.3)$ & 156.3 & 0.211 \\
\hline $\mathrm{V}_{\mathrm{z}}\left(\mathrm{L} / \mathrm{m}^{2}\right)$ & $663.8 \quad(48.1)$ & $433.4 \quad(31.1)$ & 0.040 \\
\hline $\operatorname{AUC}_{\infty}(\mathrm{ng} \cdot \mathrm{h} / \mathrm{mL})$ & $14788.6 \quad(45.3)$ & $12602.7 \quad(21.0)$ & 0.524 \\
\hline $\mathrm{C}_{\max }(\mathrm{ng} / \mathrm{mL})$ & 22968.6 (II 2.5$)$ & $3543.3 \quad(57.2)$ & $<0.001$ \\
\hline$t_{\max }(h)$ & $0.36(45.2)$ & $20.14 \quad(55.8)$ & $<0.001$ \\
\hline$t_{1 / 2}(h)$ & $21.6(17.2)$ & $20.5(14.6)$ & 0.479 \\
\hline
\end{tabular}

Data adapted from Hawkins et al (2004).

Abbreviations: $\mathrm{CL}$, total body clearance; $\mathrm{Vd}_{\mathrm{ss}}$, volume of distribution at steady state; $\mathrm{V}_{\mathrm{z}}$, volume of distribution; $\mathrm{AUC}_{\infty}$, area under the concentration-time curve from time 0 to infinity; $C_{\max }$, peak concentration; $t_{\max }$, time to peak concentration; $t_{1 / 2}$, half-life. the maximum concentration and time to maximum concentration were observed. Paclitaxel pharmacokinetic parameters including $\mathrm{AUC}_{\infty}$ and half-life were similar for the two formulations: $14789 \mathrm{ng} \cdot \mathrm{h} / \mathrm{mL}$ and 21.6 hours for ABI-007 and $12603 \mathrm{ng} \cdot \mathrm{h} / \mathrm{mL}$ and 20.5 hours for Taxol (Table 3). Plasma clearance and volumes of distribution were statistically different: $21.12 \mathrm{~L} / \mathrm{h} \cdot \mathrm{m}^{2}$ and $663.8 \mathrm{~L} / \mathrm{m}^{2}$ for ABI007 and $14.76 \mathrm{~L} / \mathrm{h} \cdot \mathrm{m}^{2}$ and $433.4 \mathrm{~L} / \mathrm{m}^{2}$ for Taxol (Hawkins et al 2004). These in vivo pharmacokinetic findings are consistent with the hypothesis that when paclitaxel is administered as Taxol, it becomes sequestered in CrEL micelles, prolonging circulation time in the intravascular space (Sparreboom et al 1999), thereby decreasing systemic clearance. The similarity of the terminal half-life of paclitaxel obtained in patients receiving CrEL-containing and CrEL-free formulations suggests that the principal effect of CrEL on paclitaxel clearance is related to changes in drug distribution rather than drug elimination.

\section{Paclitaxel metabolism and elimination}

Paclitaxel is extensively metabolized in humans, and systemic elimination of paclitaxel has been demonstrated to be saturable in vivo (Huizing et al 1993; Sonnichsen et al 1994; Rowinsky 1997). Two cytochrome P450 (CYP) isoenzymes are involved in the biotransformation of paclitaxel by human liver microsomes (Cresteil et al 1994; Harris et al 1994; Kumar et al 1994; Rahman et al 1994). The formation of the major metabolite, $6 \alpha-$ hydroxypaclitaxel, is catalyzed by CYP2C8 (Cresteil et al 1994; Rahman et al 1994), whereas the minor metabolite, p-hydroxy-phenyl-C3'-paclitaxel, is formed by CYP3A4 (Cresteil et al 1994; Harris et al 1994; Kumar et al 1994). It is believed that the dihydroxylated metabolite results from stepwise hydroxylations at the two previously described sites (Cresteil et al 1994). 
The hepatobiliary elimination of paclitaxel in a CrELcontaining formulation has been investigated using an isolated perfused rat-liver model. In this study, CrEL caused a statistically significant dose-dependent inhibition of the elimination of paclitaxel. After a dose of CrEL, a 9-fold increase in AUC, 9-fold decrease in total clearance, and 5fold increase in elimination half-life of paclitaxel were observed. Thereby, this study demonstrated the major effect of CrEL was to inhibit the hepatic elimination of paclitaxel in the isolated perfused rat liver, primarily by preventing the drug from reaching sites of metabolism and excretion (Ellis and Webster 1999). These results are consistent with other in vitro studies reporting a possible effect of CrEL on drug distribution, including decreased uptake of paclitaxel into human liver slices (Sonnichsen and Relling 1994) and by human tumor cell lines (Liebmann et al 1994).

There is also some evidence in this study that CrEL inhibited paclitaxel metabolism. In both humans and rodents, paclitaxel is eliminated primarily by microsomal hydroxylation followed by biliary excretion, with 3 '- $\mathrm{p}$ hydroxypaclitaxel being a major rat metabolite (Monsarrat et al 1990) and 6 $\alpha$-hydroxypaclitaxel being the major human metabolite (Monsarrat et al 1993). In this study the major metabolite in bile co-eluted with 3'-p-hydroxypaclitaxel, and when normalized to biliary paclitaxel concentrations the biliary excretion was decreased by CrEL. A concomitant increase in these metabolites in either perfusate or liver was not observed, suggesting that formation of these metabolites rather than excretion was inhibited.

There is little information on the effect of CrEL on microsomal metabolism. It has been reported that at clinically achievable concentrations CrEL prevented the metabolism of paclitaxel to $6 \alpha$-hydroxypaclitaxel in human liver microsomes (Jamis-Dow et al 1995). Cremophor RH40, a similar polyoxyl hydrogenated castor oil, was shown to inhibit CYP3A4-mediated nifedipine oxidation in hepatic microsomes (Wandel et al 2003). No information is currently available on the in vitro or in vivo metabolism of paclitaxel formulated as ABI-007, nor is it known how the pharmacokinetic profile of coadministered agents which are substrates for the CYP system will be affected.

\section{Paclitaxel pharmacodynamics}

Pharmacokinetic-pharmacodynamic relationships have been proposed for paclitaxel disposition and two of its major toxicities, myelosuppression (predominantly neutropenia) and neurotoxicity. The incidence of neutropenia associated with paclitaxel has been evaluated as a function of total dose,
AUC, and peak plasma concentration (ten Bokkel Huinink et al 1993), and correlation was found between these pharmacokinetic parameters and neutropenia. The observation that a 24-hour infusion schedule, at a dose of 135 or $175 \mathrm{mg} / \mathrm{m}^{2}$, is associated with a much greater degree of neutropenia is compatible with a hypothesis that neutropenia is likely to be related to the duration that the plasma paclitaxel concentrations remain at or above a "threshold" concentration (Huizing et al 1993). Gianni et al (1995) have suggested that the relationship between the length of time that plasma paclitaxel concentrations exceeded $0.05 \mu \mathrm{mol} / \mathrm{L}$ and the resulting degree of neutropenia can be well described by a sigmoid-Emax model, in which the length of time that paclitaxel concentrations remain above a given value is a function of dose, schedule, and individual patient pharmacokinetic parameters.

Neuropathy and musculoskeletal toxicities also have been related to paclitaxel pharmacokinetic parameters. Neurotoxicity in adults has been associated both with repetitive paclitaxel doses and with paclitaxel doses that exceed $250 \mathrm{mg} / \mathrm{m}^{2}$ (Rowinsky et al 1992). Additional risk factors for neurologic toxicities include prior neuropathy and prior neurotoxic chemotherapy (Donehower and Rowinsky 1993).

\section{Paclitaxel-anticancer drug interactions}

Clinically significant drug interactions with paclitaxel have been reported. Pharmacodynamic interactions with paclitaxel can be sequence or schedule dependent. Because paclitaxel undergoes hepatic oxidation via the CYP system, pharmacokinetic interactions due to enzyme induction or inhibition can also occur. Clinically significant interactions with paclitaxel have been reported for carboplatin, cisplatin, doxorubicin, epirubicin, and anticonvulsants. Administration of paclitaxel prior to doxorubicin and epirubicin results in an increase in the AUC of doxorubicin and epirubicin and enhanced toxicity (Holmes et al 1996; Esposito et al 1999; Venturini et al 2000). Conversely, paclitaxel administration following cisplatin results in an increase in myelosuppression, possibly due to deceased paclitaxel clearance (Rowinsky et al 1991). With concurrent anticonvulsant therapy, CYP enzyme induction results in decreased paclitaxel plasma steady-state concentrations (Chang et al 1998). Other drug interactions with paclitaxel have been reported in preliminary studies, but the clinical significance has not been established (Baker and Dorr 2001). 
An advantageous sequence-dependent pharmacodynamic interaction has been observed when paclitaxel is infused prior to carboplatin, resulting in decreased platelet toxicity with no effect on pharmacokinetics (Bookman et al 1996; Baker 1997; Huizing et al 1997; van Warmerdam et al 1997; Belani et al 1999; Baker and Dorr 2001). Despite no change in the pharmacokinetics of either compound, examination of the relationship between carboplatin systemic exposure and thrombocytopenia revealed that the carboplatin AUC associated with a 50\% decrease in platelet count increased from $34 \mu \mathrm{g} \cdot \mathrm{h} / \mathrm{mL}$ when carboplatin was given alone to $57 \mu \mathrm{g} \cdot \mathrm{h} / \mathrm{mL}$ when it was administered after paclitaxel (Baker 1997; Belani et al 1999). The mechanism of this interaction is not clearly understood.

\section{Clinical experience with ABI-007}

In the first phase I clinical trial in patients with advanced or refractory cancer, 19 patients received doses of ABI-007 ranging from 135 to $375 \mathrm{mg} / \mathrm{m}^{2}$ given as a 30 -minute intravenous infusion every 3 weeks, without any premedication (Ibrahim et al 2002). As expected, no hypersensitivity reactions were observed during the infusion period, and hematological toxicity was mild and not cumulative. The dose-limiting toxicity, which occurred in three of six patients treated at $375 \mathrm{mg} / \mathrm{m}^{2}$, consisted of sensory neuropathy, stomatitis, and superficial keratopathy. The maximum tolerated dose at that administration schedule was determined to be $300 \mathrm{mg} / \mathrm{m}^{2}$.

The maximum tolerated dose of ABI-007 given on a weekly basis has also been determined. In a phase I study, 39 patients with advanced non-hematologic malignancies received doses of ABI-007 ranging from 80 to $200 \mathrm{mg} / \mathrm{m}^{2} /$ day as a 30 -minute infusion once a week for 3 weeks followed by a week of rest (Nyman et al 2004). Patients were divided into two groups, those who had been heavily pretreated with prior chemotherapy and those who had not. The maximum tolerated dose of ABI-007 was $100 \mathrm{mg} / \mathrm{m}^{2} /$ week in patients who had previously been heavily pretreated, with the dose-limiting toxicity of grade 4 neutropenia. In the group of patients who had not been heavily pretreated, grade 3 peripheral neuropathy was the dose-limiting toxicity at $150 \mathrm{mg} / \mathrm{m}^{2} /$ week. Five patients, all of whom had prior therapy with paclitaxel, demonstrated a partial response. This study provides evidence that ABI007 can be given safely on a weekly schedule.

Two phase II trials of ABI-007, using an every-3-week administration regimen have been conducted in patients with metastatic breast cancer. In the first trial, 43 patients were treated with ABI-007 at a dose of $175 \mathrm{mg} / \mathrm{m}^{2}$ (Ibrahim et al 2002). The adverse event profile was similar to that described for the phase I daily dosing study, with mild hematological toxicity, no allergic reactions, and no grade 3 or 4 non-hematological toxicity other than vomiting in $2 \%$ of patients. Three patients had complete responses and 18 achieved partial responses for an overall objective response rate of $51 \%$ (Ibrahim et al 2002). In the second trial, 63 patients were treated at a dose of $300 \mathrm{mg} / \mathrm{mL}$. Neutropenia was noted, as well as peripheral neuropathy grades $3 / 4$ in $10 \%$ of patients, myalgia in $5 \%$, vomiting in $2 \%$, diarrhea in $3 \%$, rash in $2 \%$, and amblyopia in $2 \%$, but none developed hypersensitivity reactions. Three patients had complete responses, and 34 achieved partial responses, for an overall objective response rate of $61 \%$.

ABI-007 was compared against the standard paclitaxel formulation Taxol in a phase III trial in patients with metastatic breast cancer (O'Shaughnessy et al 2003). This study randomized 454 patients to either ABI-007 $260 \mathrm{mg} / \mathrm{m}^{2}$ infused over 30 minutes once every 3 weeks without premedication or Taxol $175 \mathrm{mg} / \mathrm{m}^{2}$ administered over 3 hours once every 3 weeks with premedication, including dexamethasone and antihistamines. Patients were randomized in a 1:1 fashion and were required to have measurable disease. Patients previously treated with a taxane were excluded; however, $78 \%$ of patients had been exposed to prior anthracyclines. Approximately $40 \%$ of patients received treatment on trial as first-line therapy, $40 \%$ as second-line therapy, and approximately $20 \%$ as third-line therapy or greater.

In this phase III study, patients treated with ABI-007 achieved a significantly higher objective response rate and time to progression than those treated with Taxol. Based on an investigator-scored assessment, the overall response rate for ABI-007 was 33\% compared with $19 \%$ for Taxol $(p=0.001)$ with a median time to progression of 21.9 weeks for ABI-007 versus 16.1 weeks for Taxol $(p=0.03)$. A blinded independent radiology review was conducted and the objective response rate for all patients treated with Taxol was $10 \%$ vs $21 \%$ among patients treated with ABI-007 $(p=0.002)$. While overall response rate was lower in both the ABI-007 and paclitaxel arms according to the blinded independent radiology review as compared with an investigator-scored assessment, treatment with ABI-007 resulted in a significantly superior response compared with paclitaxel formulated in CrEL.

Consistent with the early phase studies, there was no incidence of grade $3 / 4$ hypersensitivity reactions in the group 
of patients treated with ABI-007, despite the absence of premedication. Grade 4 neutropenia was significantly reduced in patients treated with ABI-007 occurring in only $9 \%$ of patients compared with $22 \%$ treated with Taxol $(p=0.001)$. Grade 3 sensory neuropathy was higher in the ABI-007 arm (10\%) than in the Taxol arm (2\%), with no episodes of grade 4 neuropathy. However, sensory neuropathy was improved to grades 1 or 2 within a median of 22 days if ABI-007 was withheld. Flushing occurred more frequently in patients treated with Taxol $(5 \%)$ than in those treated with ABI-007 (less than 1\%).

\section{Conclusion and future investigations}

Paclitaxel is an anticancer agent that has been shown to be effective in the treatment of a variety of solid tumors; however, owing to its insolubility paclitaxel is formulated with the micelle-forming vehicle CrEL, which results in increased toxicity and altered pharmacokinetics of paclitaxel. ABI-007 is a CrEL-free, albumin-stabilized, nanoparticle paclitaxel formulation that has been shown to be free from infusion-related hypersensitivity reactions, which allows higher paclitaxel doses to be administered over a shorter infusion duration. Pharmacokinetic studies indicate that in contrast to CrEL-containing paclitaxel, ABI-007 displays linear pharmacokinetics over the clinically relevant dose range of $135-300 \mathrm{mg} / \mathrm{m}^{2}$. In a phase III study conducted in patients with metastatic breast cancer, patients treated with ABI-007 achieved a significantly higher objective response rate and time to progression than those treated with CrEL-containing paclitaxel.

When paclitaxel is given concurrently with other drugs, pharmacokinetic and pharmacodynamic interactions may occur. Because paclitaxel is extensively metabolized by hepatic CYP3A4 and CYP2C8, drugs that are metabolized by, inhibit, or induce these enzymes have the potential to alter paclitaxel clearance. The pharmacodynamics of drugs given in combination with paclitaxel can also be altered; this may depend on the sequence or schedule of administration and can yield both favorable and undesirable results. Many of the pharmacokinetic and pharmacodynamic interactions with paclitaxel have been described in studies employing CrEL-containing paclitaxel. Therefore, the effect of paclitaxel administered as ABI-007 needs to be assessed.

Clinical data suggest that use of this nanoparticle albumin-bound paclitaxel formulation may enable clinicians to administer paclitaxel at higher doses with less toxicity than is seen with CrEL-containing paclitaxel. It is essential to relate the pharmacokinetics of paclitaxel to both the toxic and therapeutic outcomes resulting from its use so that optimal dosing strategies can be utilized. As the development of ABI-007 moves forward it will be important to assess the effect of this delivery vehicle on the pharmacokinetics of paclitaxel when it is administered in combination with other drugs. Clinical trials investigating the role of ABI007 in combination with other antineoplastic agents are warranted.

\section{References}

Adams JD, Flora KP, Goldspiel BR, et al. 1993. Taxol: a history of pharmaceutical development and current pharmaceutical concerns. $J$ Natl Cancer Inst Monogr, (15):141-7.

Baker AF, Dorr RT. 2001. Drug interactions with the taxanes: clinical implications. Cancer Treat Rev, 27:221-33.

Baker SD. 1997. Drug interactions with the taxanes. Pharmacotherapy, 17(5 Pt 2):126S-132S.

Belani CP, Kearns CM, Zuhowski EG, et al. 1999. Phase I trial, including pharmacokinetic and pharmacodynamic correlations, of combination paclitaxel and carboplatin in patients with metastatic non-small-cell lung cancer. J Clin Oncol, 17:676-84.

Bookman MA, McGuire WP, Kilpatrick D, et al. 1996. Carboplatin and paclitaxel in ovarian carcinoma: a phase I study of the Gynecologic Oncology Group. J Clin Oncol, 14:1895-902.

Brouwer E, Verweij J, De Bruijn P, et al. 2000. Measurement of fraction unbound paclitaxel in human plasma. Drug Metab Dispos, 28: $1141-5$.

Chang SM, Kuhn JG, Rizzo J, et al. 1998. Phase I study of paclitaxel in patients with recurrent malignant glioma: a North American Brain Tumor Consortium report. J Clin Oncol, 16:2188-94.

Cresteil T, Monsarrat B, Alvinerie P, et al. 1994. Taxol metabolism by human liver microsomes: identification of cytochrome P450 isozymes involved in its biotransformation. Cancer Res, 54:386-92.

Desai N, Trieu V, Yao R, et al. 2004. Increased transport of nanoparticle albumin-bound paclitaxel (ABI-007) by endothelial gp60-mediated caveolar transcytosis: a pathway inhibited by Taxol. Eur $J$ Cancer Suppl, 2:182.

Desai N, Yao Z, Soon-Shiong P, et al. 2002. Evidence of enhanced in vivo efficacy at maximum tolerated dose (MTD) of nanoparticle paclitaxel (ABI-007) and taxol in 5 human tumor xenografts of varying sensitivity to paclitaxel. Proc Am Soc Clin Oncol, 21:462.

Desai N, Yao Z, Trieu V, et al. 2003. Evidence of greater tumor and red cell partitioning and superior antitumor activity of Cremophor free nanoparticle paclitaxel (ABI-007) compared to Taxol. Proc Am Soc Clin Oncol, 22:156 (abstract 625).

Donehower RC, Rowinsky EK. 1993. An overview of experience with TAXOL (paclitaxel) in the USA. Cancer Treat Rev, 19 Suppl C: 63-78.

Ellis AG, Webster LK. 1999. Inhibition of paclitaxel elimination in the isolated perfused rat liver by Cremophor EL. Cancer Chemother Pharmacol, 43:13-18.

Esposito M, Venturini M, Vannozzi MO, et al. 1999. Comparative effects of paclitaxel and docetaxel on the metabolism and pharmacokinetics of epirubicin in breast cancer patients. J Clin Oncol, 17:1132.

Garber K. 2004. Improved paclitaxel formulation hints at new chemotherapy approach. J Natl Cancer Inst, 96:90-1.

Gelderblom H, Mross K, ten Tije AJ, et al. 2002. Comparative pharmacokinetics of unbound paclitaxel during 1- and 3-hour infusions. J Clin Oncol, 20:574-81.

Gelderblom H, Verweij J, Nooter K, et al. 2001. Cremophor EL: the drawbacks and advantages of vehicle selection for drug formulation. Eur J Cancer, 37:1590-8 
Gianni L, Kearns CM, Giani A, et al. 1995. Nonlinear pharmacokinetics and metabolism of paclitaxel and its pharmacokinetic/ pharmacodynamic relationships in humans. J Clin Oncol, 13:180-90.

Gianni L, Vigano L, Locatelli A, et al. 1997. Human pharmacokinetic characterization and in vitro study of the interaction between doxorubicin and paclitaxel in patients with breast cancer. J Clin Oncol, 15:1906-15

Harris JW, Rahman A, Kim BR, et al. 1994. Metabolism of taxol by human hepatic microsomes and liver slices: participation of cytochrome P450 3A4 and an unknown P450 enzyme. Cancer Res, 54:4026-35.

Hawkins MJ, Lane JR, Harris L, et al. 2004. Comparative pharmacokinetic (PK) study of a Cremophor-free, protein stabilized, nanoparticle formulation (ABI-007) and a Cremophor-based formulation of paclitaxel (P) in patients with advanced solid tumors. Eur J Cancer Suppl, 2:164.

Henningsson A, Karlsson MO, Vigano L, et al. 2001. Mechanism-based pharmacokinetic model for paclitaxel. J Clin Oncol, 19:4065-73.

Holmes FA, Madden T, Newman RA, et al. 1996. Sequence-dependent alteration of doxorubicin pharmacokinetics by paclitaxel in a phase I study of paclitaxel and doxorubicin in patients with metastatic breast cancer. J Clin Oncol, 14:2713-21.

Huizing MT, Giaccone G, van Warmerdam LJ, et al. 1997. Pharmacokinetics of paclitaxel and carboplatin in a dose-escalating and dose-sequencing study in patients with non-small-cell lung cancer. The European Cancer Centre. J Clin Oncol, 15:317-29.

Huizing MT, Keung AC, Rosing H, et al. 1993. Pharmacokinetics of paclitaxel and metabolites in a randomized comparative study in platinum-pretreated ovarian cancer patients. J Clin Oncol, 11: 2127-35.

Ibrahim NK, Desai N, Legha S, et al. 2002. Phase I and pharmacokinetic study of ABI-007, a Cremophor-free, protein-stabilized, nanoparticle formulation of paclitaxel. Clin Cancer Res, 8:1038-44.

Ibrahim NK, Samuels B, Page R, et al. 2002. Nanoparticle paclitaxel (ABI007) in metastatic breast cancer (MBC): efficacy and evidence of dosedependent activity in two multicenter phase II studies. Proc Am Soc Clin Oncol, 21:209.

Jamis-Dow CA, Klecker RW, Katki AG, et al. 1995. Metabolism of Taxol by human and rat liver in vitro: a screen for drug interactions and interspecies differences. Cancer Chemother Pharmacol, 36:107-14.

John TA, Vogel SM, Tiruppathi C, et al. 2003. Quantitative analysis of albumin uptake and transport in the rat microvessel endothelial monolayer. Am J Physiol Lung Cell Mol Physiol, 284:L187-96.

Karlsson MO, Molnar V, Freijs A, et al. 1999. Pharmacokinetic models for the saturable distribution of paclitaxel. Drug Metab Dispos, 27:1220-3.

Kearns CM. 1997. Pharmacokinetics of the taxanes. Pharmacotherapy, 17(5 Pt 2):105S-109S.

Kumar GN, Walle UK, Walle T. 1994. Cytochrome P450 3A-mediated human liver microsomal taxol 6 alpha-hydroxylation. J Pharmacol Exp Ther, 268:1160-5.

Liebmann J, Cook JA, Lipschultz C, et al. 1994. The influence of cremophor EL on the cell cycle effects of paclitaxel (Taxol) in human tumor cell lines. Cancer Chemother Pharmacol, 33:331-9.

Loos WJ, Szebeni J, ten Tije AJ, et al. 2002. Preclinical evaluation of alternative pharmaceutical delivery vehicles for paclitaxel. Anticancer Drugs, 13:767-75.

Monsarrat B, Alvinerie P, Wright M, et al. 1993. Hepatic metabolism and biliary excretion of Taxol in rats and humans. $J$ Natl Cancer Inst Monogr, 15:39-46.

Monsarrat B, Mariel E, Cros S, et al. 1990. Taxol metabolism. Isolation and identification of three major metabolites of taxol in rat bile. Drug Metab Dispos, 18:895-901.

Mross K, Hollander N, Hauns B, et al. 2000. The pharmacokinetics of a 1-h paclitaxel infusion. Cancer Chemother Pharmacol, 45:463-70.

Nuijen B, Bouma M, Schellens JH, et al. 2001. Progress in the development of alternative pharmaceutical formulations of taxanes. Invest New Drugs, 19:143-53.
Nyman DW, Campbell KJ, Hersh E, et al. 2004. A phase I trial of ABI007, nanoparticle paclitaxel, administered to patients with advanced non-hematologic malignancies. Proc Am Soc Clin Oncol, 23:133.

O'Shaughnessy J, Tjulandin S, Davidson N, et al. 2003. ABI-007 $\left(\mathrm{ABRAXANE}^{\mathrm{TM}}\right)$, a nanoparticle albumin-bound (nab) paclitaxel demonstrates superior efficacy vs taxol in MBC: a phase III trial. Proceedings from the 26th annual San Antonio Breast Cancer Symposium. 2003 Dec 3-6; San Antonio, Texas, USA (abstract 44).

Rahman A, Korzekwa KR, Grogan J, et al. 1994. Selective biotransformation of Taxol to 6 alpha-hydroxytaxol by human cytochrome P450 2C8. Cancer Res, 54:5543-6.

Rowinsky EK. 1997. The development and clinical utility of the taxane class of antimicrotubule chemotherapy agents. Аnпи Rev Med, 48: $353-74$.

Rowinsky EK, Gilbert MR, McGuire WP, et al. 1991. Sequences of taxol and cisplatin: a phase I and pharmacologic study. J Clin Oncol, 9: 1692-703.

Rowinsky EK, Onetto N, Canetta RM, et al. 1992. Taxol: the first of the taxanes, an important new class of antitumor agents. Semin Oncol, 19:646-62.

Sonnichsen DS, Hurwitz CA, Pratt CB, et a1. 1994. Saturable pharmacokinetics and paclitaxel pharmacodynamics in children with solid tumors. J Clin Oncol, 12:532-8.

Sonnichsen DS, Relling MV. 1994. Clinical pharmacokinetics of paclitaxel. Clin Pharmacokinet, 27:256-69.

Sparreboom A, van Asperen J, Mayer U, et al. 1997. Limited oral bioavailability and active epithelial excretion of paclitaxel (Taxol) caused by P-glycoprotein in the intestine. Proc Natl Acad Sci U S A, 94:2031-5

Sparreboom A, van Tellingen O, Nooijen WJ, et al. 1996a. Nonlinear pharmacokinetics of paclitaxel in mice results from the pharmaceutical vehicle Cremophor EL. Cancer Res, 56:2112-15.

Sparreboom A, van Tellingen O, Nooijen WJ, et al. 1996b. Tissue distribution, metabolism and excretion of paclitaxel in mice. Anticancer Drugs, 7:78-86.

Sparreboom A, van Zuylen L, Brouwer E, et al. 1999. Cremophor ELmediated alteration of paclitaxel distribution in human blood: clinical pharmacokinetic implications. Cancer Res, 59:1454-7.

Sykes E, Woodburn K, Decker D, et al. 1994. Effects of Cremophor EL on distribution of Taxol to serum lipoproteins. Br J Cancer, 70:401-4.

ten Bokkel Huinink WW, Eisenhauer E, Swenerton K. 1993. Preliminary evaluation of a multicenter, randomized comparative study of TAXOL (paclitaxel) dose and infusion length in platinum-treated ovarian cancer. Canadian-European Taxol Cooperative Trial Group. Cancer Treat Rev, 19 Suppl C:79-86.

Terwogt JM, Nuijen B, Huinink WW, et al. 1997. Alternative formulations of paclitaxel. Cancer Treat Rev, 23:87-95.

van Warmerdam LJ, Huizing MT, Giaccone G, et al. 1997. Clinical pharmacology of carboplatin administered in combination with paclitaxel. Semin Oncol, 24(1 Supp1 2):S2-97-S2-104.

van Zuylen L, Gianni L, Verweij J, et al. 2000. Inter-relationships of paclitaxel disposition, infusion duration and Cremophor EL kinetics in cancer patients. Anticancer Drugs, 11:331-7.

van Zuylen L, Karlsson MO, Verweij J, et al. 2001. Pharmacokinetic modeling of paclitaxel encapsulation in Cremophor EL micelles. Cancer Chemother Pharmacol, 47:309-18.

van Zuylen L, Verweij J, Sparreboom A. 2001. Role of formulation vehicles in taxane pharmacology. Invest New Drugs, 19:125-41.

Venturini M, Lunardi G, Del Mastro L, et al. 2000. Sequence effect of epirubicin and paclitaxel treatment on pharmacokinetics and toxicity. J Clin Oncol, 18:2116-25.

Wandel C, Kim RB, Stein CM. 2003. "Inactive" excipients such as Cremophor can affect in vivo drug disposition. Clin Pharmacol Ther, 73:394-6.

Wani MC, Taylor HL, Wall ME, et al. 1971. Plant antitumor agents. VI. The isolation and structure of taxol, a novel antileukemic and antitumor agent from Taxus brevifolia. J Am Chem Soc, 93:2325-7. 